\title{
Acanthamoeba Adherence to Contact Lenses and Removal by Cleaning Agents
}

\author{
S. KILVINGTON ${ }^{1}$ and D. F. P. LARKIN ${ }^{2}$ \\ Bath and Bristol
}

\begin{abstract}
Summary
The quantitative adherence of a keratitis isolate of Acanthamoeba polyphaga to lowand high-water content non-ionic soft contact lenses and one type of rigid gaspermeable lens was investigated. Adherence of trophozoite and cyst forms of the organism was observed in vitro, and adherent amoebae counted by a plaque assay method following detachment. Trophozoites adhered to all lens types with adherence being statistically significantly greater to high water content soft lenses. Cyst attachment occurred only to the soft lenses but not to gas-permeable lenses, and was significantly higher for the high water content lenses. Attachment of cysts was significantly lower than that of trophozoites to each lens tested. Recommended cleaning procedures using two commercial solutions removed all adherent trophozoites and cysts from lenses. These studies demonstrate

(i) that lenses may act as a vector in Acanthamoeba keratitis, particularly for highwater content lenses and trophozoite amoebae,

(ii) that lens cleaning agents may prevent keratitis by removing adherent Acanthamoeba.
\end{abstract}

Acanthamoeba is a genus of free-living amoebae found in most soil and aquatic environments. ${ }^{1}$ Keratitis caused by Acanthamoeba is significantly linked with wear of contact lenses; especially soft lenses. ${ }^{2}$ Acanthamoeba have been isolated from the lens storage cases of many patients in whom Acanthamoeba keratitis has been diagnosed. ${ }^{3}$ Acanthamoeba contaminating a storage case might be transmitted to the cornea by adhering to the lens. Increasing incidence of Acanthamoeba keratitis, especially in wearers of soft lenses, prompted us to study adherence of this amoeba to lenses.

It has been reported that amoebae may adhere to soft contact lenses. ${ }^{4}$ We used a quantitative method to study soft lenses of dif- fering water content and a rigid gas-permeable lens in order to ascertain whether preferential adherence might explain higher incidence of infection in wearers of certain lens types. New lenses were studied, using trophozoite and cyst forms of a clinical isolate of $A$. polyphaga. In addition we tested the efficacy of lens cleaning solutions in removing the adherent amoebae. Demonstration and quantification of Acanthamoeba trophozoite and cyst adherence to lens surfaces, and their removal by recommended cleaning agents has prompted this report.

\section{Materials and Methods}

Test Organism

A strain of $A$. polyphaga (Shinh) which had

From: ${ }^{2}$ Department of Ophthalmology, Bristol Eye Hospital, Bristol and ${ }^{1}$ Public Health Laboratory, Royal United Hospital, Bath.

Correspondence to: Mr D. F. P. Larkin, Moorfields Eye Hospital, City Road, London EC1V 2PD. 


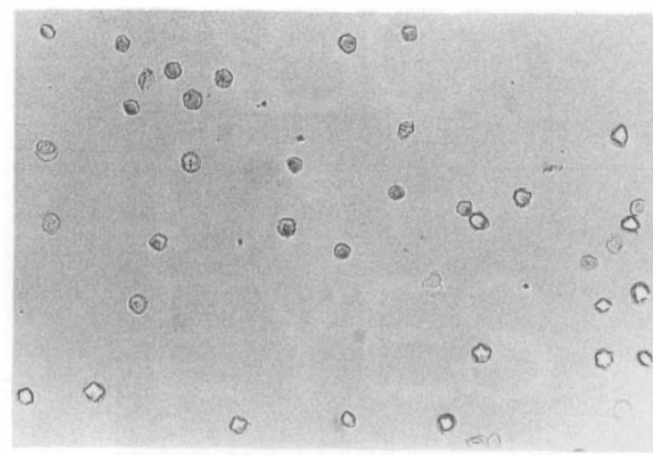

Fig. 1. Acanthamoeba cysts adherent to a lidofilcon lens, following immersion in cyst suspension for four hours and prior to detachment by addition of ice-cold saline and agitation $(\times 200)$.

been isolated from a keratitis patient was used in this study (kindly given by Dr. David Warhurst, London School of Hygiene and Tropical Medicine). The strain was adapted to axenic culture in a serum-casein-glucoseyeast extract medium, ${ }^{5}$ modified here by the inclusion of $0.1 \%$ filter-sterilized Panmede liver digest (Paines and Byrne Ltd, Greenford, England). Trophozoites were maintained in tissue culture flasks at $32^{\circ} \mathrm{C}$. Mature cysts were produced from these cultures using the constant $\mathrm{pH}$ encystment medium of $\mathrm{Neff}^{\mathrm{t}}$ in stationary tissue culture flasks incubated in air at $32^{\circ} \mathrm{C}$.

\section{Lenses}

Three types of contact lenses were studied:

(a) soft extended-wear lidofilcon A (70\% water, 30\% non-ionic lidofilcon A [poly (nvinyl pyrrolidone + methylmethacrylate) with allyl methacrylate and ethylene dimethacrylate]),

(b) soft daily-wear polymacon (38.6\% water, $61.4 \%$ non-ionic polymacon [2-hydroxyethyl methacrylate cross-linked with ethylene glycol dimethacrylate])

(c) rigid gas-permeable sulphylcon A (fluorosilicon-acrylate).

Lenses were stored in sterile vials in normal saline at room temperature until used.

\section{Adherence to lens materials}

Immediately prior to testing, the trophozoites or cysts were washed three times with normal saline. Cell counts were performed using a haemocytometer and a concentration of
$1 \times 10^{5} / \mathrm{ml}$ prepared. Three lenses of each type were placed into individual wells of a 24 well tissue culture plate (Nunc Tissue Culture Products, Gibco BRL Ltd., Paisley, United Kingdom) and $1 \mathrm{ml}$ of the calibrated trophozoite or cyst suspension added. Trophozoite attachment was allowed to occur for two hours and cyst attachment for four hours, at room temperature. The culture plate was stationary during this time and the process was monitored using light microscopy.

Lenses were then removed and rinsed twice in separate $10 \mathrm{ml}$ volumes of normal saline. Amoebae remaining adherent (Figs. 1 and 2) were then detached by adding the lenses to $10 \mathrm{ml}$ of ice cold normal saline and placing in ice for 15 minutes. At five minute intervals, the lenses were agitated on a vortex mixer for ten seconds. This treatment caused complete detachment of both trophozoites and cysts, verified by microscopic observation of the lens surface. Serial ten fold dilutions of the saline solution were made in Page's amoebal saline (PAS) ${ }^{1}$ and $1 \mathrm{ml}$ volumes plated out in triplicate on to the surface of nutrient agar plates $(2.5 \%$ agar, $0.1 \%$ glucose and $0.1 \%$ yeast extract in PAS). Before use, the plates had been seeded with live Escherichia coli using a sterile swab moistened in a turbid súspension of the bacterium in PAS. Plates inoculated with the test dilutions were allowed to dry at room temperature before incubation in a humidified incubator at $35^{\circ} \mathrm{C}$ for up to seven days. Under these conditions, $E$. coli divided to form a confluent lawn on the plate with zones of clearing, termed plaques, resulting from growth of individual trophozoites, or hatched cysts.

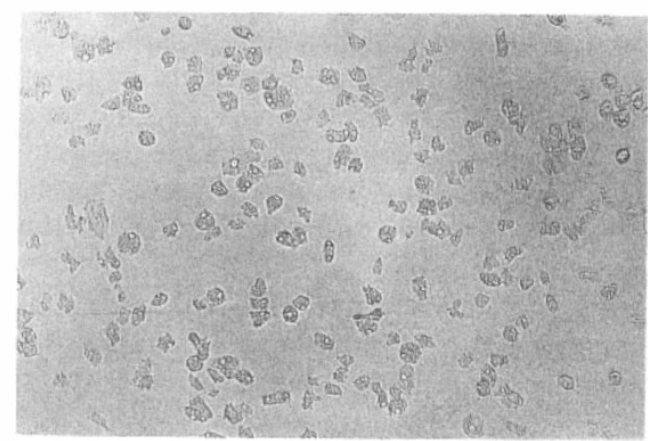

Fig. 2. Trophozoites adherent to a lidofilcon lens following immersion in trophozoite suspension for two hours $(\times 100)$. 
Table I Acanthamoeba polyphaga cyst and trophozoite adherence to contact lenses.

\begin{tabular}{llllllll}
\hline Amoeba & $\begin{array}{l}\text { Lens } \\
\text { material }\end{array}$ & $\begin{array}{l}\text { water } \\
\text { content } \\
(\%)\end{array}$ & Undiluted & $10^{-1}$ & $10^{-2}$ & $10^{-3}$ & $10^{-4}$ \\
\hline cysts & lidofilcon A & 70 & $9.3 \pm 2.0$ & $1.2 \pm 0.6$ & 0 & - & - \\
& polymacon & 38.6 & $6.1 \pm 2.0$ & $0.3 \pm 0.1$ & 0 & - & - \\
& sulphylcon A & - & 0 & 0 & 0 & - & - \\
trophs & lidofilcon A & 70 & - & - & $>15$ & $>15$ & $5.0 \pm 1.7$ \\
& polymacon & 38.6 & - & - & $>15$ & $7.9 \pm 2.4$ & $0.4 \pm 0.1$ \\
& sulphylcon A & - & - & - & $>15$ & $7.0 \pm 1.6$ & $0.3 \pm 0.1$ \\
\hline
\end{tabular}

Values given are mean \pm standard deviation.

Significantly more cysts adhered to lidofilcon than polymacon $(\mathrm{t}=3.25 ; 2$-tailed $\mathrm{t}$-test; $\mathrm{p}=0.005)$.

More trophozoites adhered to lidofilcon than polymacon $(t=6.94 ; p<0.001)$. The difference between adherence of trophozoites to polymacon and gas-permeable (sulphylcon $\mathrm{A})$ was not significant $(\mathrm{t}=0.87 ; \mathrm{p}=0.4)$.

The degree of attachment of amoebae to a lens surface was expressed as the number of plaque-forming units (PFU)/ml. A one-way analysis of variance (ANOVA $)^{7}$ was carried out simultaneously on the results of adherence to all three lenses to assess overall differences. Then a two-tailed t-test was used in analysis and the level of significance taken as $5 \%(\mathrm{p}<0.05)$.

\section{Efficacy of Cleaning}

The efficacy of two contact lens cleaning solutions in removing Acanthamoeba adherent to lenses was investigated. Trophozoites or cysts were attached to the lens surfaces as described above. Cyst adherence to gas-permeable lenses was found only to occur when c. $10^{3}$ cysts were air dried on to the lens surface. The lenses were then cleaned according to the solution manufacturer's instructions using

(i) $10 \%$ sodium tridecylether sulphate ("Cleaner for rigid gas-permeable lenses", Bausch and Lomb, Middlesex, England) for the gas-permeable lenses

(ii) EDTA and sorbic acid ("Daily Cleaner for soft contact lenses", Bausch and Lomb) for the lidofilcon and polymacon lenses.

Following one rinse in $10 \mathrm{ml}$ of normal saline, the lenses were examined for the presence of remaining amoebae using light microscopy, with phase contrast and magnification $\times 100$.

\section{Results}

\section{Adherence to lens materials}

Counts of adherent trophozoites and cysts are shown in Table I. Three counts were obtained from each lens tested, and three lenses of each type were studied. From the nine counts, a mean and standard deviation was calculated for each lens type.

One-way ANOVA showed a significant overall difference in adherence to all lenses for trophozoites $(\mathrm{p}<0.001)$ and cysts $(\mathrm{p}<0.001)$. A 2-tailed t-test indicated significantly greater adherence, after two hours, of trophozoites to lidifilcon than to polymacon lenses $(t=6.94 ; \mathrm{df}=16 ; \mathrm{p}<0.001)$. No significant difference was observed between adherence to polymacon and gas-permeable lenses $(t=0.87 ; \mathrm{df}=16 ; \mathrm{p}=0.4)$.

Significantly greater adherence, after four hours, of cysts to lidofilcon than to polymacon lenses was observed $(t=3.25 ; \quad \mathrm{df}=16$; $\mathrm{p}=0.005)$. No cysts were observed to adhere to gas-permeable lenses.

\section{Efficacy of Cleaning}

No trophozoites or cysts were observed to remain attached to the surface of the lenses following cleaning and rinsing using the solution manufacturer's instructions.

\section{Discussion}

We found adherence of substantial numbers of Acanthamoeba trophozoites to each lens type. Acanthamoeba trophozoite adherence is significantly higher to high water-content lidofilcon lenses than low water-content polymacon or gas-permeable lenses. We found that cyst adherence to lidofilcon was also substantially higher than to polymacon lenses, and that no cysts remained adherent to gaspermeable lenses. The difference in water 
content ( $70 \%$ and $38.6 \%)$ may alone account for the differential soft lens adherence, both lenses being composed of non-ionic polymers. However lens surface characteristics may also be a factor: lidofilcon $\mathrm{A}$ lenses are lathe-cut and polymacon lenses spun cast. It is interesting to note that studying adherence of Pseudomonas aeruginosa to a number of soft lenses of varying hydration, Miller and Ahearn ${ }^{8}$ found no strict correlation between adherence and percent hydration but found that slightly more bacteria adhered to lidofilcon than polymacon lenses. Other workers have found decreased bacterial adherence with increased water content. ${ }^{9-11}$

It has been shown that adherence of bacteria is facilitated by mucin ${ }^{12}$ or protein ${ }^{11}$ coating of lenses and that bacteria preferentially adhere to organic debris and lens scratches. ${ }^{13}$ These factors may also influence Acanthamoeba adherence, and it is likely that study of new lenses underestimates the adherence which occurs in vivo. Studies of bacterial adherence to contact lenses have included electron microscopy of sectioned soft lens fragments. ${ }^{8,14,15}$ The difficulty in cutting hard lenses prompted us to compare adherence of both lens types by quantitative plaque assay, a technique ideally suited to study of Acanthamoeba.

Acanthamoeba keratitis has been increasingly recognised in recent years and contact lens wear is the predominant risk factor. Epidemiological study of 208 cases in the United States indicated that $160(85 \%)$ wore contact lenses, of which $89(56 \%)$ wore daily-wear soft, $31(19 \%)$ wore extended-wear soft, and $14(9 \%)$ wore hard or gas-permeable lenses. ${ }^{2}$ These data may reflect the popularity of, and numbers wearing different lens types. The data may also reflect the role of the soft lens material in disease pathogenesis. In any event, it is probable that daily-wear lens users inoculate Acanthamoeba from contaminated lens storage cases to the cornea each day: these patients therefore have a higher probability of developing keratitis than those using extended-wear lenses, irrespective of surface adherence characteristics.

Contamination of lens solutions and storage cases is a major risk factor in Acanthamoeba keratitis. ${ }^{3}$ Contact lens storage cases provide an environment which can readily be contaminated by Acanthamoeba. ${ }^{16}$ This is one reason why so many Acanthamoeba keratitis patients are lens wearers. Our results demonstrate another reason: Acanthamoeba trophozoites and cysts can adhere to lenses, which are a suitable vehicle for transfer of contaminating amoebae to the cornea. It is possible that a further factor is lens-induced epithelial trauma providing an entry route for the Acanthamoeba to the stroma, but the necessity of an epithelial break in induction of keratitis remains uncertain.

Whether in vivo a loose or a tight adhesion to the lens allows greater numbers of amoebae to be inoculated on the cornea is uncertain. The effects of lid movement or the tear film on adherence here demonstrated in vitro remain uncertain. Tightly adherent Acanthamoeba will not be washed away by lid movement or the tear film, but may not transfer to the cornea and cause keratitis.

Stern and $\mathrm{Zam}^{17}$ have demonstrated that effective enzymatic cleaning agents remove mucin coating of lenses such that the adherence of Ps. aeruginosa is inhibited. The complete removal of Acanthamoeba from lenses by use of lens cleaning agents in this study indicates that these compounds are effective for this purpose also. Clinical impressions suggest that many lens wearers default on regular use of disinfectant and cleaning agents. This report demonstrates a role for cleaning agents in prevention of Acanthamoeba keratitis.

We thank Dr JP Leeming, Department of Microbiology, Bristol Royal Infirmary for assistance with statistical analysis. Lenses and solutions were kindly donated by Bausch and Lomb, Middlesex, England.

References

'Page FC: An illustrated key to freshwater and soil amoebae. Scientific publication No. 34. Ambleside. Freshwater Biological Association, 1976.

${ }^{2}$ Stehr-Green JK, Bailey TM, Visvesvara GS: The epidemiology of Acanthamoeba keratitis in the United States. Am J Ophthalmol 1989, 107: 331-6.

${ }^{3}$ Moore MB, McCulley JP, Newton C, et al.: Acanthamoeba keratitis. A growing problem in soft and 
hard contact lens wearers. Ophthalmology 1987. 94: 1654-61.

${ }^{+}$Lindquist TD, Doughman DJ, Rubenstein JB, Moore JW, Campbell RC: Acanthamoeba-contaminated hydrogel contact lenses. Susceptibility to disinfection. Cornea 1988, 7: 300-3.

${ }^{5}$ Aufy S, Kilvington S, Mann PG, Warhurst DC: Improved selective isolation of Naegleria fowleri from the environment. Trans Roy Soc Trop Med Hyg 1986, 80: 350-1.

${ }^{6}$ Neff RJ, Ray SA, Benton WF, Wilborn M: Induction of synchronous encystment (differentiation) in Acanthamoeba sp. Methods in Cell Physiol 1964, 1: $55-83$.

${ }^{7}$ Bourke GJ, Daly LE, McGilvray J: Interpretation and uses of medical statistics 3rd ed. Oxford, Blackwell, 1985, 124-8.

${ }^{8}$ Miller MJ and Ahearn DG: Adherence of Pseudomonas aeruginosa to hydrophilic contact lenses and other substrata. J Clin Microbiol 1987, 25: 1392-7.

${ }^{9}$ Dart JK and Badenoch PR: Bacterial adherence to contact lenses. CLAO J 1986, 12: 220-4.

${ }^{10}$ Pringle JH, Fletcher M: Influence of substratum hydration and adsorbed macromolecules on bacterial attachment to surfaces. Appl Environ Microbiol 1983, 51: 1321-5.
${ }^{11}$ Miller MJ, Wilson LA, Ahearn DG: Effects of protein, mucin and human tears on adherence of Pseudomonas aeruginosa to hydrophilic contact lenses. J Clin Microbiol 1988, 26: 513-7.

"Stern GA and Zam ZS: The pathogenesis of contact lens-associated Pseudomonas aeruginosa corneal ulceration: I. The effect of contact lens coatings on adherence of Pseudomonas aeruginosa to soft contact lenses. Cornea 1986, 5: 41-5.

${ }^{13}$ Tripathi RC, Tripathi BJ, Ruben M: The pathology of soft contact lens spoilage. Ophthalmology 1980, 87: 365-80.

${ }^{14}$ Duran JA, Refojo MF, Gipson IK, Kenyon KR: Pseudomonas attachment to new hydrogel contact lenses. Arch Ophthalmol 1987, 105: 106-9.

${ }^{15}$ Slusher MM, Myrvik QN, Lewis JC, Gristina AG: Extended-wear lenses, biofilm, and bacterial adhesion. Arch Ophthalmol 1987, 105: 110-5.

${ }^{16}$ Larkin DFP, Kilvington S, Easty DL: Contamination of contact lens storage cases by Acanthamoeba and bacteria. Br J Ophthalmol 1990, 74: 133-5.

${ }^{17}$ Stern GA and Zam ZS: The effect of enzymatic contact lens cleaning on adherence of Pseudomonas aeruginosa to soft contact lenses. Opthalmology 1987, 94: 115-9. 